\title{
Review: terazosin improves urological symptoms in benign prostatic hyperplasia
}

Wilt TJ, Howe RW,Rutks IR, et al. Terazosin for benign prostatic hyperplasia. Cochrane Database Syst Rev 2002;(4):CD003851 (latest version 14 Sep 1999).

\section{QUESTION: In men with benign prostatic hyperplasia (BPH), does the non-uroselective $\alpha$ blocker terazosin improve urological symptoms?}

\section{Data sources}

Studies were identified by searching Medline (19662001), the Cochrane Library, the Prostatic Diseases and Urologic Malignancies Group specialised register; and reviewing bibliographies of relevant studies and reviews.

\section{Study selection}

Studies were selected if they were randomised controlled trials (RCTs) in any language comparing terazosin with placebo, phytotherapy, or pharmacological or surgical therapies in men with symptomatic BPH and had a treatment duration $\geq 4$ weeks.

\section{Data extraction}

2 reviewers independently extracted data on methodological quality, study design, patient characteristics, enrolment criteria, outcomes, adverse effects, and dropouts. The main outcome was change in urological symptoms measured by validated symptom scores (Boyarsky Symptom Score, American Urological Association [AUA], and International Prostate Symptom Score [IPSS]) (AUA and IPSS scales are identical), presented as the percentage absolute improvement from baseline.

\section{Main results}

17 RCTs (5151 men, mean age $65 \mathrm{y}$ ) met the inclusion criteria. 10 RCTs compared terazosin with placebo, 7 with other $\alpha$ blockers, 1 with finasteride (also had a placebo group), and 1 with transurethral microwave thermotherapy. Study durations ranged from 4-52 weeks. A random effects model was used to pool results where possible. Urinary symptom scores improved more in men who received terazosin than in those who received placebo (6 RCTs). The pooled mean percentage improvement in urinary symptoms using the Boyarsky Symptom Score was greater in those who received terazosin (4 RCTs, 37\% v 15\%). In 2 RCTs that investigated the effect of terazosin withdrawal on BPH symptoms, symptom scores were better in men who continued to receive terazosin $(15-25 \%)$ than in men who withdrew from terazosin and received placebo (41$63 \%)$. In 4 of 5 flexible dose escalation trials, symptom improvement was greater with terazosin (31-38\%) than placebo (10-18\%). 1 RCT compared terazosin with finasteride: for improvement in AUA symptom scores, terazosin was better than finasteride $(38 \% v 20 \%)$ and similar to terazosin and finasteride combined $(38 \% v$ $39 \%)$. Terazosin was similar in urological symptom improvement to tamsulosin (4 RCTs), alfuzosin (1 RCT), doxazosin (1 RCT), and prazosin (1 RCT). Terazosin did not show as much improvement from baseline in the IPSS as did transurethral microwave thermotherapy (1 RCT, $42 \%$ v 65\%)

\section{Conclusions}

In men with benign prostatic hyperplasia, terazosin improves urological symptoms more than placebo or finasteride and is similar in effectiveness to other $\alpha$ blockers. 1 trial comparing terazosin with transurethral microwave thermotherapy showed terazosin to be less effective.

\section{COMMENTARY}

$\mathrm{BPH}$ is a common problem for older men that can adversely affect quality of life. The rigorous literature synthesis by Wilt et al provides convincing evidence that terazosin helps men with moderately symptomatic BPH. Terazosin was more effective than placebo or finasteride in reducing symptom scores and improving peak urinary flow. Combination treatment with terazosin and finasteride was no more effective than terazosin alone. However, finasteride, a 5 - $\alpha$ reductase inhibitor that can shrink prostate tissue, may still be useful for men with very large ( $>60 \mathrm{ml}$ ) prostates. ${ }^{1}$ In addition, finasteride, unlike terazosin, has been shown to reduce the probability of surgery and acute urine retention. ${ }^{2}$

All $\alpha$ blockers improved urological symptoms. However, terazosin, which inhibits vascular $\alpha$ adrenergic receptors, often caused orthostatic hypotension, dizziness, and asthaenia; $6-24 \%$ of study patients withdrew for adverse events. Tamsulosin, the only uroselective $\alpha$-1 blocker, caused less vasodilatation and fewer than $1 \%$ of patients withdrew for adverse events. However, the studies reviewed used $0.2 \mathrm{mg}$ doses of tamsulosin; side effects occur more frequently with the prescription strength doses $(0.4-0.8 \mathrm{mg})$ available in the US. ${ }^{3}$

Terazosin was less effective than transurethral microwave thermotherapy, a minimally invasive urological procedure for BPH that is not widely available. Terazosin would probably be even less effective than transurethral resection of the prostate (TURP), the gold standard treatment for BPH that can reduce symptom scores by $66 \%$ and improve peak urinary flow by $54 \%{ }^{4}$ However, TURP requires hospital admission, and treatment complications are common. Terazosin is a relatively effective and safe alternative for managing moderately symptomatic BPH in primary care practice. Richard M Hoffman, MD MPH New Mexico VA Health Care System Albuquerque, New Mexico, USA

1 Walsh PC. Treatment of benign prostatic hyperplasia. NEngl IMed 1996:335:586-7.

2. Med 1996;335:586-7. $\mathrm{R}$. Walsh $\mathrm{P}$ et al The effect of finasteride on the Bisk of acte, Wist $\mathrm{P}$, alen. for .

3 Wilt TJ, MacDonald R, Rutks I. Tamsulosin for benign prostatic hyperplasia. Cochrane Database Syst Rev 003:(1):CD002081.

4 Wasson JH, Reda DJ, Bruskewitz RC, et al. A comparison of ransurethral surgery with watchful waiting for moderate symptoms of benign prostatic hyperplasia. $N$ Engl J Med 995;332:75-9.
Source of funding: Department of Veteran Affairs Health Services Research and Development Service.

For correspondence: Dr TJ Wilt, Minneapolis VA Center for Chronic Disease Outcomes Research, Minneapolis, MN, USA. Tim.Wilt@med.va.gov

Abstract and commentary also appear in ACP Journal Club. 